\title{
Métodos de aplicação e inseticidas no controle de percevejos na cultura da soja
}

\section{Application methods and insecticides for controlling stink bugs in soybean}

\author{
Rubens Alex Fiorin'; Glauber Renato Stürmer²; Jerson Vanderlei Carús Guedes ${ }^{3 *}$; \\ Ivan Francisco Dressler da Costa ${ }^{4}$; Clérison Régis Perini ${ }^{5}$
}

\section{Resumo}

A aplicação de inseticidas é a forma mais utilizada no controle de percevejos na cultura da soja, sendo a escolha da tecnologia de aplicação e do inseticida fundamental para obtenção de um controle eficiente. O experimento foi desenvolvido em lavoura comercial de soja, na safra agrícola 2008/09, em São Sepé, RS, com o objetivo de avaliar a eficiência de três métodos de aplicação e dois inseticidas no controle de percevejos na soja. O delineamento experimental utilizado foi inteiramente casualizado, em esquema fatorial $3 \times 2+1$ testemunha, com cinco repetições. As unidades experimentais tiveram dimensões de 40,0 x 40,0 m. O fator A foi representado pelos métodos de aplicação, sendo: A1- Terrestre com pontas hidráulicas, A2- Aérea, com atomizadores rotativos de discos e A3- Aérea, com pontas hidráulicas. O fator B foi representado pelos inseticidas sendo: B1- tiametoxam + lambda-cialotrina e B2- acefato. As aplicações terrestres e aéreas com baixo volume oleoso apresentam maior eficiência no controle de percevejos na cultura da soja, refletindo-se este controle em maior rendimento de grãos. Os inseticidas tiametoxam + lambda-cialotrina e acefato apresentam controle de percevejos e efeito residual semelhante. Palavras-chave: Controle químico, baixo volume oleoso, pentatomidae

\begin{abstract}
Insecticides application is the most widely used form to control stink bugs in soybean, being the application technology and the insecticide used the major factors for an efficient control. The experiment was conducted in a soybean field during the 2008/09 growing season, in São Sepé, RS, in order to evaluate efficiency of three methods of application and two insecticides for controlling stink bugs in soybeans. The experimental design used was completely randomized, in a factorial scheme $3 \times 2+1$ control, with five replicates. The experimental units had dimensions of 40.0 x $40.0 \mathrm{~m}$. The factor A was represented by the methods of application, as follows: A1- Tractorized spray with hydraulic nozzle; A2Aerial, with rotary disc atomizers and A3- Aerial, with hydraulic nozzles. The factor B was represented by the insecticides, as follows: B1- thiamethoxam + lambda-cyhalothrin and B2-acephate. Ground and aerial applications with low volume oily had greater efficiency for controlling stink bugs in soybean, where led to an increase in soybean grain yield. Insecticides thiamethoxam + lambda-cyhalothrin and acephate were similar in controlling stink bugs and in residual effect.
\end{abstract}

Key words: Chemical control, low volume oily, pentatomidae

${ }^{1}$ Eng $^{\mathrm{o}}$ Agr $^{\mathrm{o}}$ Msc., Universidade Federal de Santa Maria, UFSM. E-mail: rubensfiorin@yahoo.com.br

2 Aluno do Programa de Pós-graduação em Agronomia, Universidade Federal de Santa Maria, UFSM. E-mail: glautec@yahoo. com.br

3 Prof. Dr., Departamento de Defesa Fitossanitária, Universidade Federal de Santa Maria, UFSM/CCR/DFS, Prédio 42, sala 3225, Av. Roraima, 1000, CEP:97105-900, Santa Maria, RS. E-mail: jerson.guedes@gmail.com

4 Prof. Dr., Departamento de Defesa Fitossanitária, Universidade Federal de Santa Maria. E-mail: ifdresler@gmail.com

5 Aluno do Curso de Agronomia, Universidade Federal de Santa Maria. E-mail: periniagro@gmail.com

Autor para correspondência 


\section{Introdução}

Entre os insetos pragas da cultura da soja destacam-se os percevejos, que sugam preferencialmente os legumes atingindo diretamente os grãos da soja (CORRÊA-FERREIRA; PANIZZI, 1999; CORRÊA-FERREIRA, 2005). Esse ataque causa perdas significativas no rendimento, através da diminuição do peso dos grãos, abortamento dos legumes, perdas na qualidade e no potencial germinativo da soja (GAZZONI, 1998; CORREAAFERREIRA; AZEVEDO, 2002). No Brasil ocorrem principalmente as espécies Nezara viridula (Linneaus, 1758), Piezodorus guildinii (Westwood, 1837) e Euchistus heros (Fabricius, 1794), estando a predominância de uma espécie relacionada, principalmente, à temperatura (SILVA; CORRÊAFERREIRA; SOSA-GOMÉZ, 2006) e a extensão dos danos, dependente do estádio de desenvolvimento das plantas (BELORTE et al., 2003).

O método de manejo de percevejos mais utilizado é o controle químico, através da pulverização de inseticidas, tornando a escolha do produto e da tecnologia utilizada fundamentais, uma vez que a eficiência biológica da pulverização é dependente não somente de produtos com ação comprovada, mas também da tecnologia empregada na sua aplicação (CARVALHO; FURLANI JUNIOR, 1999; BALAN et al., 2005).

De acordo com Ozeki e Kunz (1998) a cobertura do alvo é o principal fator responsável pela eficiência da aplicação, tornando a escolha da ponta de pulverização, fundamental para obtenção do espectro de gotas adequado. O momento de aplicação é outro fator que deve ser considerado na tomada de decisão (CUNHA et al., 2008).

De acordo com Teixeira (1997) as pulverizações são realizadas predominantemente com pontas hidráulicas, via aérea ou terrestre. Para Monteiro (2005), as pontas hidráulicas de aplicação podem ser substituídas por outros sistemas de formação de gotas, como atomizadores rotativos de discos $\left(\mathrm{BVO}^{\circledR}\right)$, que formam uma neblina mais homogênea, proporcionada pelo controle do tamanho das gotas e maior eficiência biológica.

Considerando a importância dos percevejos para a cultura da soja e a necessidade de informações a respeito da tecnologia de aplicação de inseticidas para seu controle, esse trabalho objetivou avaliar a eficiência de controle de três tecnologias de aplicação e dois inseticidas no controle de percevejos na cultura da soja.

\section{Material e Métodos}

O experimento foi conduzido em lavoura comercial de soja, em São Sepé, RS, no ano agrícola 2008/09. A soja, cultivar CD 214 RR, foi semeada em 15 de novembro, com espaçamento de $0,45 \mathrm{~m}$ entre linhas, na densidade de 400 mil plantas ha ${ }^{-1}$. A adubação, controle de plantas daninhas, pragas iniciais, lagartas e demais tratos culturais foram realizados seguindo as Indicações técnicas para a cultura da soja (EMBRAPA, 2006).

O delineamento experimental utilizado foi inteiramente casualizado, em esquema fatorial $3 \times 2+1$ testemunha, com cinco repetições. As unidades experimentais mediam 40,0 $\mathrm{m}$ x 40,0 m. O fator $A$ foi representado pelos métodos de aplicação, sendo: A1- Terrestre com pontas hidráulicas (BHD-T), A2- Aérea, com atomizadores rotativos de discos (ARD-A) e A3- Aérea, com bicos hidráulicos (BHD-A). O fator B foi representado pelos inseticidas sendo: B1- tiametoxam + lambdacialotrina $\left(28,2+21,2\right.$ g i.a. ha $\left.^{-1}\right)$ e B2- acefato $(225,0$ g i.a. ha-1 $)$.

A aplicação terrestre foi realizada com pulverizador tratorizado Jacto Condor com barra de $12,0 \mathrm{~m}$, equipada com 24 bicos hidráulicos e pontas BJ 110.01. A pressão de trabalho foi de 60 psi, velocidade de aplicação de $6 \mathrm{~km} \mathrm{~h}^{-1}$, barra de aplicação posicionada a $0,40 \mathrm{~m}$ do topo da cultura e volume de aplicação de $120 \mathrm{~L} \mathrm{ha}^{-1}$. Segundo indicações do fabricante (JACTO MÁQUINAS AGRÍCOLAS S/A, 2010) essa ponta produz gotas com indução de ar e de diâmetro variável de 350 a 
700 micrômetros $(\mu \mathrm{m})$, gotas grossa a muito grossa de acordo com a norma ASAE S-572.

Para realização das aplicações aéreas utilizouse um avião agrícola Air Tractor. Na aplicação do tratamento $\mathrm{A} 2$, utilizou-se atomizadores rotativos de discos (Turboaero $^{\circledR}$ ) modelo TA-88C-5-8, com pás fixadas na posição 3 , pressão de trabalho de 30 psi, altura de vôo de 4,0 m, faixa de deposição efetiva de $18,0 \mathrm{~m}$ e volume de aplicação de $12 \mathrm{~L} \mathrm{ha}^{-}$ 1. O veículo utilizado foi água + óleo vegetal marca Agróleo $^{\circledR}\left(0,5 \mathrm{~L} \mathrm{ha}^{-1}\right)$. De acordo com Monteiro (2006) atomizadores rotativos de discos aplicando em baixo volume e adição de óleo adjuvante produz gotas de diâmetro na faixa de 80 a $200 \mu \mathrm{m}$, gotas muito finas a média de acordo com a norma ASAE S-572.

Para o tratamento $\mathrm{A} 3$, com bicos hidráulicos foram utilizadas pontas D6, core 45, operando com pressão de 38 psi, altura de vôo de 3,0 m, faixa de deposição efetiva de $16,0 \mathrm{~m}$ e volume de aplicação de $20 \mathrm{~L} \mathrm{ha}^{-1}$. De acordo com Monteiro (2006) estes bicos hidráulicos, produzem gotas de diâmetro na faixa de 200 a $300 \mu \mathrm{m}$, gotas grossas de acordo com classificação constante na norma ASAE S-572.

Durante a aplicação a umidade relativa do ar manteve-se em $60 \% \pm 3$, a temperatura em $27^{\circ} \mathrm{C} \pm$ 2 e velocidade do vento de 3 a $4 \mathrm{~km} \mathrm{~h}^{-1}$.

A eficiência de controle foi avaliada através de amostragens utilizando pano-de-batida, contabilizando conjuntamente todas as espécies de percevejos adultos e ninfas grandes $\left(3^{\circ}\right.$ a $5^{\circ}$ instar). Foram realizadas 10 amostragem por unidade experimental, sendo uma prévia (antes da pulverização) e aos 3, 7, 11 e 15 dias após a aplicação dos tratamentos (DAT).

Visando quantificar a redução no rendimento ocasionada pelos insetos, foi realizada a determinação do rendimento de grãos, através da colheita de cinco amostras por parcela, totalizando uma área útil de $3,7 \mathrm{~m}^{2}$ que posteriormente foram degranados e pesados. Para determinação do peso de 1000 grãos foram contabilizadas quatro repetições de cada amostra.

Os dados foram transformados por $\sqrt{x+0,5}$ as análises foram feitas utilizando o software ESTAT. Os dados originais foram submetidos à análise da variância e as médias comparadas pelo teste Tukey a $5 \%$ de probabilidade de erro. A eficiência dos tratamentos foi calculada através da equação de Abbott (1925).

\section{Resultados e Discussão}

$\mathrm{Na}$ avaliação prévia à aplicação dos tratamentos a população de percevejos apresentou média de 4,25 indivíduos (adultos e ninfas grandes) por amostra, satisfatória à instalação do experimento por estar acima do nível de controle de quatro percevejos/ amostra, para lavouras de produção de grãos (HOFFMANN-CAMPO et al., 2000). Não houve interação do fator inseticida com as tecnologias de aplicação avaliadas. Assim, os inseticidas tiametoxam + lambda-cialotrina $(28,2+21,2 \mathrm{~g}$ i.a. ha $\left.{ }^{-1}\right)$ e acefato $\left(225,0\right.$ g i.a. ha $\left.{ }^{-1}\right)$ responderam de forma similar à tecnologia de aplicação, exercendo controle semelhante em todas as datas de amostragens (Tabela 1).

Tabela 1. Número de percevejos (média) por pano-de-batida, em resposta à aplicação de inseticidas na cultura da soja. Safra 2008/09, São Sepé, RS.

\begin{tabular}{lcccccc}
\hline \multirow{2}{*}{\multicolumn{1}{c}{ Inseticidas }} & \multirow{2}{*}{$\begin{array}{c}\text { Dosagens } \\
\end{array}$} & $\left(\mathrm{g} \mathrm{i.a.} \mathrm{ha}^{-1}\right)$ & Prévia & \multicolumn{5}{c}{ Dias após a aplicação } \\
\cline { 3 - 6 } & & \multicolumn{5}{c}{ Número de insetos em $0,90 \mathrm{~m}^{2}$} \\
\hline Tiam.+ Lambda* & $28,2+21,2$ & $4,15^{\text {ns }}$ & $1,06 \mathrm{~b}$ & $1,10 \mathrm{~b}$ & $1,18 \mathrm{~b}$ & $0,86 \mathrm{~b}$ \\
Acefato & 225,0 & 4,29 & $1,10 \mathrm{~b}$ & $1,24 \mathrm{~b}$ & $1,16 \mathrm{~b}$ & $0,96 \mathrm{~b}$ \\
Testemunha & - & 4,27 & $3,76 \mathrm{a}$ & $3,90 \mathrm{a}$ & $4,24 \mathrm{a}$ & $3,01 \mathrm{a}$ \\
\hline CV $(\%)$ & - & 18,46 & 25,1 & 24,8 & 30,35 & 30,14 \\
\hline
\end{tabular}

*Tiam. + Lambda $=$ Tiametoxam + lambda-cialotrina. ${ }^{\text {ns }}$ Diferença não significativa pelo teste Tukey a $5 \%$ de probabilidade de erro. 
Comparando-se as tecnologias de aplicação (Tabela 2), verifica-se que aos três dias após a aplicação dos tratamentos (DAT) a pulverização terrestre resultou em um controle superior aos tratamentos com aplicação aérea. Entretanto, aos sete DAT verificou-se a menor população de percevejos nas aplicações ARD-A e BHD-T, em relação à aplicação aérea com BHD-A. Segundo Furlan
(2005), a maior eficiência das aplicações realizadas com ARD-A ocorre, devido ao sistema produzir um espectro de gotas com menor diâmetro e mais uniforme, com maior capacidade de penetração no interior do dossel, em comparação com o espectro de gotas produzidas por BHD. Nas avaliações realizadas aos 11 e 15 DAT, não foram observadas diferenças significativas no controle proporcionado pelas diferentes tecnologias de aplicação.

Tabela 2. Número de percevejos (média) por pano-de-batida, em resposta a aplicação de inseticidas com diferentes tecnologias de aplicação na cultura da soja. Safra 2008/09, São Sepé, RS.

\begin{tabular}{lccccc}
\hline \multirow{2}{*}{$\begin{array}{c}\text { Tecnologias de } \\
\text { aplicação }\end{array}$} & Prévia & 3 & \multicolumn{5}{c}{ Dias após a aplicação } \\
\cline { 2 - 5 } & \multicolumn{5}{c}{ Número de insetos em $0,90 \mathrm{~m}^{2}$} \\
\hline BHD-T* & $4,17^{\mathrm{ns}}$ & $0.91 \mathrm{c} * *$ & $1,09 \mathrm{c}$ & $1,05 \mathrm{~b}$ & $0,96 \mathrm{~b}$ \\
ARD-A & 4,25 & $1,10 \mathrm{~b}$ & $1,08 \mathrm{c}$ & $1,18 \mathrm{~b}$ & $0,91 \mathrm{~b}$ \\
BHD-A & 4,34 & $1,35 \mathrm{~b}$ & $1,45 \mathrm{~b}$ & $1,27 \mathrm{~b}$ & $1,08 \mathrm{~b}$ \\
Testemunha & 4,27 & $3,76 \mathrm{a}$ & $3,90 \mathrm{a}$ & $4,24 \mathrm{a}$ & $3,01 \mathrm{a}$ \\
\hline CV $(\%)$ & 18,40 & 35,15 & 24,88 & 30,35 & 30,14 \\
\hline
\end{tabular}

* BHD-T $=$ Terrestre $\left(120 \mathrm{~L} \mathrm{ha}^{-1}\right)$; ARD-A = Aérea com atomizadores rotativos de discos $\left(12 \mathrm{~L} \mathrm{ha}^{-1}\right)$ e BHD-A = Aérea com bico hidráulico $\left(20 \mathrm{~L} \mathrm{ha}^{-1}\right)$.

**Médias não seguidas pelas mesmas letras nas colunas diferem entre si pelo teste Tukey a 5\% de probabilidade de erro.

${ }^{n}$ Diferença não significativa pelo teste Tukey a 5\% de probabilidade de erro.

O efeito dos tratamentos sobre a população de percevejos é apresentado na (Tabela 3), com destaque as maiores reduções ocasionada pela aplicação dos inseticidas via terrestre, com volume de calda de $120 \mathrm{~L} \mathrm{ha}^{-1}$ e com atomizadores rotativos de disco adicionado de óleo adjuvante, com taxa de aplicação de $12 \mathrm{~L} \mathrm{ha}^{-1}$.

Tabela 3. Número de percevejos por pano-de-batida, em resposta a aplicação de inseticidas com diferentes tecnologias de pulverização na cultura da soja. Safra 2008/09, São Sepé, RS.

\begin{tabular}{lcccc}
\hline \multirow{2}{*}{ Tratamentos } & \multicolumn{4}{c}{ Dias após a aplicação } \\
\cline { 2 - 5 } & 3 & 7 & 11 & 15 \\
\hline Tiam.+ Lambda BHD-T* & $0,34 \mathrm{c} * *$ & $0,40 \mathrm{c}$ & $0,81 \mathrm{c}$ & $0,62 \mathrm{c}$ \\
Acefato BHD-T & $0,50 \mathrm{c}$ & $0,52 \mathrm{c}$ & $0,71 \mathrm{c}$ & $0,41 \mathrm{c}$ \\
Tiam.+ Lambda ARD-A & $0,71 \mathrm{bc}$ & $0,61 \mathrm{c}$ & $0,91 \mathrm{~b}$ & $0,31 \mathrm{c}$ \\
Acefato ARD-A & $1,10 \mathrm{~b}$ & $0,90 \mathrm{bc}$ & $1,11 \mathrm{~b}$ & $0,62 \mathrm{c}$ \\
Tiam.+ Lambda BHD-A & $1,52 \mathrm{~b}$ & $1,53 \mathrm{~b}$ & $1,41 \mathrm{~b}$ & $0,62 \mathrm{c}$ \\
Acefato BHD-A & $1,11 \mathrm{~b}$ & $1,30 \mathrm{~b}$ & $1,10 \mathrm{~b}$ & $1,03 \mathrm{~b}$ \\
Testemunha & $3,76 \mathrm{a}$ & $3,90 \mathrm{a}$ & $4,24 \mathrm{a}$ & $3,01 \mathrm{a}$ \\
\hline CV $(\%)$ & 26,96 & 23,1 & 18,52 & 21,64 \\
\hline
\end{tabular}

*Tiam. + Lambda $=$ Tiametoxam + lambda-cialotrina; BHD-T $=$ Terrestre $\left(120 \mathrm{~L} \mathrm{ha}^{-1}\right)$; ARD-A = Aérea com atomizadores rotativos de discos $\left(12 \mathrm{~L} \mathrm{ha}^{-1}\right)$ e BHD-A = Aérea com bico hidráulico $\left(20 \mathrm{~L} \mathrm{ha}^{-1}\right)$.

**Médias não seguidas pelas mesmas letras nas colunas diferem entre si pelo teste Tukey a 5\% de probabilidade de erro. 
Eficiência de controle superior a $80 \%$ foi observada nos tratamentos com pulverização terrestre e na aplicação com ARD-A utilizando o inseticida tiametoxam + lambda-cialotrina aos 3, 7 e 15 DAT e as menores eficiências de controle nos tratamentos aplicados com BHD-A (Tabela 4). A maior eficiência obtida pela pulverização com ARD-A, em relação à aplicação com BHD-A justifica-se pela diferença no espectro de gotas produzido por cada um dos sistemas de pulverização.
Enquanto os bicos hidráulicos produzem gotas, desde muito finas até muito grossas, os atomizadores rotativos são capazes de produzir espectro mais homogêneo de gotas finas, com maior capacidade de cobrir alvo e penetrar no interior do dossel. Também a adição do óleo vegetal a calda utilizada neste sistema de aplicação confere maior capacidade de espalhamento e absorção e reduz a tensão superficial da folha, podendo determinar o nível de retenção das gotas e influenciar na eficiência biológica da pulverização (MENDONÇA, 2003).

Tabela 4. Eficiência de controle (\%), peso de 1000 grãos (g), rendimento de grãos (em kg ha-1 e \%). Safra 2008/09, São Sepé, RS.

\begin{tabular}{|c|c|c|c|c|c|c|}
\hline \multirow{2}{*}{ Tratamentos } & \multicolumn{3}{|c|}{ Dias após a aplicação } & \multirow[b]{2}{*}{15} & \multirow[t]{2}{*}{ Peso 1000 grãos } & \multirow{2}{*}{$\begin{array}{l}\text { Rendimento } \\
\mathrm{Kg} \mathrm{ha}^{-1}\end{array}$} \\
\hline & 3 & 7 & 11 & & & \\
\hline Tiam.+ Lambda BHD-T* & $91,92 * *$ & 89,74 & 80,95 & 80,37 & $158,7 \mathrm{bc} * * *$ & $2362,20 \mathrm{a}$ \\
\hline Acefato BHD-T & 86,49 & 86,66 & 83,33 & 86,29 & $155,71 \mathrm{c}$ & $2389,83 \mathrm{a}$ \\
\hline Tiam.+ Lambda ARD-A & 81,08 & 84,61 & 78,57 & 89,65 & $164,58 \mathrm{a}$ & $2356,26 \mathrm{a}$ \\
\hline Acefato ARD-A & 70,27 & 76,92 & 73,81 & 79,31 & $156,23 \mathrm{c}$ & $2264,18 a b$ \\
\hline Tiam.+ Lambda BHD-A & 59,46 & 61,53 & 66,66 & 79,31 & $160,56 \mathrm{~b}$ & $2157,33 \mathrm{bc}$ \\
\hline Acefato BHD-A & 70,27 & 66,66 & 73,88 & 65,51 & $164,01 \mathrm{a}$ & $2177,89 \mathrm{bc}$ \\
\hline Testemunha & 0,00 & 0,00 & 0,00 & 0,00 & $154,44 \mathrm{~cd}$ & $1992,57 d$ \\
\hline CV $(\%)$ & - & - & - & - & 9,91 & 16,4 \\
\hline
\end{tabular}

Tiam. + Lambda $=$ Tiametoxam + lambda-cialotrina; BHD-T $=$ Terrestre $\left(120 \mathrm{~L} \mathrm{ha}^{-1}\right)$; ARD-A = Aérea com atomizadores rotativos de discos $\left(12 \mathrm{~L} \mathrm{ha}^{-1}\right)$ e BHD-A = Aérea com bico hidráulico $\left(20 \mathrm{~L} \mathrm{ha}^{-1}\right)$.

** Calculada pela formula de Abbot (1925).

*** Médias não seguidas pelas mesmas letras nas colunas diferem entre si pelo teste Tukey a 5\% de probabilidade de erro.

A importância do tamanho das gotas cresce em função da maior dificuldade de alcance do alvo, sendo a parte inferior das plantas local de refúgio as pragas. Os diâmetros das gotas formadas são diferentes nos diversos equipamentos para pulverização, buscando-se maior homogeneidade no tamanho das gotas, sendo que o bico de pulverização é o elemento que mais influencia neste parâmetro (BLANCA, 1999).

$\mathrm{O}$ rendimento de grãos refletiu a eficiência de controle dos tratamentos aplicados com bico hidráulico terrestre e baixo volume oleoso. Ambos apresentaram diferenças significativas em relação aos demais tratamentos, indicando a necessidade de controle e a capacidade dos percevejos causarem danos à cultura, nas populações observadas (Tabela 4). Avaliando os danos causados por percevejos Villas Boas et al. (1990) observaram redução de $300 \mathrm{~kg} \mathrm{ha}^{-1}$ quando a soja foi submetida ao ataque de quatro percevejos $\mathrm{m}^{-2}$, resultado semelhante ao obtido no presente trabalho, em que a testemunha apresentou um rendimento $396,64 \mathrm{~kg} \mathrm{ha}^{-1}$ inferior ao rendimento obtido na aplicação de acefato BHD-T, em função do controle exercido pelo referido tratamento e pela permanência de populações variáveis de 3,01 a 4,27 percevejos $\mathrm{m}^{-2}$.

Observando o peso médio de 1000 grãos (Tabela 4) destaca-se a importância de adoção de medidas 
de controle do complexo de percevejos que atacam a cultura, uma vez que todos os tratamentos com aplicação de inseticidas diferiram estatisticamente da testemunha. O maior rendimento de grãos nos tratamentos que não apresentaram os maiores pesos médios de 1000 grãos pode ser explicado, de acordo com Corrêa-Ferreira e Panizzi (1999) e por Bonato (2000), por menores índices de abortamento de legumes no início de enchimento dos grãos em função do ataque de percevejos, com maior número de legumes por planta.

O controle propiciado pelos inseticidas possibilitou a diferenciação da testemunha, ressaltando a importância da adoção de medidas de controle nas populações de percevejos, porém é necessário avaliação biológica das pulverizações realizadas e ampliar a discussão quanto ao método empregado para manutenção do potencial de controle do inseticida.

\section{Conclusões}

A aplicação terrestre e aérea em baixo volume oleoso com atomizadores rotativos de discos é mais eficiente que a aplicação aérea com bico hidráulico no controle de percevejos na cultura da soja.

Os inseticidas tiametoxam + lambda-cialotrina e acefato apresentam controle de percevejos e efeito residual semelhante.

A maior eficiência de controle dos tratamentos com bico hidráulico terrestre e do inseticida tiametoxam + lambda-cialotrina aplicado em baixo volume oleoso com atomizadores rotativos de discos refletiu-se no rendimento de grãos da soja.

\section{Referências}

ABBOTT, W. S. A method of computing the effectiveness of an insecticide. Journal of Economic Entomology, Lanham, v. 18, n. 1, p. 265-267, 1925.

BALAN, M. G.; ABI-SAAB, O. J. G.; SILVA, C. G.; RIO, A. Pulverização em alvos artificiais: avaliação com o uso do software conta-gotas. Ciência Rural, Santa Maria, v. 35, n. 4, p. 916-919, 2005.

BELORTE, L. C.; RAMIRO, Z. A.; FARIA, A. M.; MARINO, C. A. B. Danos causados por percevejos (Hemiptera: Pentatomidae) em cinco cultivares de soja (Glycine max (L.) Merrill, 1917) no município de Araçatuba, SP. Arquivos Instituto Biológico, Campinas, v. 70, n. 2, p. 169-175, 2003.

BLANCA, A. L. Maquinaria agrícola: constituición, funcionamiento, regulación y cuidados. 3. ed. Madrid: Ministério da Agricultura, Pesca e Alimentação, 1999. $361 \mathrm{p}$.

BONATO, R. B. Estresses em soja. Passo Fundo: EMBRAPA Trigo, 2000. 254 p.

CARVALHO, W. P. A.; FURLANI JUNIOR, J. A. Estudo comparativo entre coletores para determinação do DMV e coeficiente de dispersão na amostragem de gotas em aplicações de produtos líquidos. Energia na Agricultura, Botucatu, v. 12, n. 1, p. 28-37, 1999.

CORRÊA-FERREIRA, B. S.; PANIZZI, A. R. Percevejos da soja e seu manejo. Londrina: EMBRAPA-CNPSo, 1999. 45 p. (EMBRAPA-CNPSo. Circular Técnica, 24).

CORRÊA-FERREIRA, B. S. Suscetibilidade da soja a percevejos na fase anterior o desenvolvimento das vagens. Pesquisa Agropecuária Brasileira, Brasília, v. 40, n. 11, p. 1067-1072, 2005.

CORRÊA-FERREIRA, B. S.; AZEVEDO, J. Soybean seed damage by different species of stink bugs. Agricultural and Forest Entomology, Londres, v. 4, n. 2, p. 145-150, 2002.

CUNHA, J. P. A. R.; MOURA, E. A. C.; SILVA JUNIOR, J. L.; ZAGO, F. A.; JULIATTI, F. C. Efeito de pontas de pulverização no controle químico da ferrugem da soja. Engenharia Agrícola, Jaboticabal, v. 28, n. 2, p. 283-291, 2008.

EMPRESA BRASILEIRA DE PESQUISA AGROPECUÁRIA - EMBRAPA. Tecnologia de produção de soja: Paraná - 2007. Londrina: Embrapa Soja, 2006. (Sistemas de produção, n. 10).

FURLAN, H. S. Impacto, diagnose e manejo da ferrugem asiática da soja no Brasil. In: REUNIÃO ITINERANTE DE FITOSSANIDADE DO INSTITUTO BIOLÓGICO, 11.; ENCONTRO DE FITOSSANIDADE DE PLANTIO DIRETO NA PALHA DO CLUBE AMIGOS DA TERRA DE AGUAÍ, 1., 2005, Aguaí. Anais... Aguaí: CAT, 2005. p. 31-32.

GAZZONI, D. L. Efeito de populações de percevejos na produtividade, qualidade da semente e características 
agronômicas da soja. Pesquisa Agropecuária Brasileira, Brasília, v. 33, n. 8, p. 1229-1237, 1998.

HOFFMANN-CAMPO, C. B.; FLÁVIO MOSCARDI; F.; CORRÊA-FERREIRA, B. S.; OLIVEIRA; L. J.; SOSA-GÓMEZ; D. R.; PANIZZI; A. R.; CORSO; I. C.; GAZZONI; D. L.; OLIVEIRA; E. B. de. Pragas da soja no Brasil e seu manejo integrado. Londrina: EmbrapaCNPso, 2000. p. 70 p. (Circular Técnica EMBRAPACNPSo),

JACTO MÁQUINAS AGRICOLAS S/A. Disponível em: <http://www.jacto.com.br>. Acesso em: 07 jul. 2010.

MENDONÇA, C. G. Efeito de óleos minerais e vegetais nas propriedades físico-quimicas das caldas de pulverização e suas interações com superficies foliares. 2003. Tese (Doutorado em Agronomia) -Universidade Estadual Paulista Julio de Mesquita Filho, Botocatu, 2003 .

MONTEIRO, M. V. de M. Curso de coordenadores em aviação agrícola. Sorocaba: Centro Brasileiro de Bioaeronáutica, 2005. 278p.
Eficiência na aplicação de defensivos com BVO aéreo. In: BORGES, L. D. Tecnologias de aplicação de defensivos agrícolas. Passo Fundo: Plantio Direto Eventos, 2006. cap. 8, p. 99-108.

OZEKI, Y.; KUNZ, R. P. Tecnologia de aplicação aérea aspectos práticos. In: GUEDES, J. V. C.; DORNELLES, S. H. B. Tecnologia e segurança na aplicação de agrotóxicos. Santa Maria: Departamento de Defesa Fitossanitária; Sociedade de Agronomia de Santa Maria, 1998. p. 65-78.

SILVA, M. T. B.; CORRÊA-FERREIRA, B. S.; SOSAGOMÉZ, D. R. Erro e resistência. Revista Cultivar Grandes Culturas, Pelotas, v. 8, n. 82, p. 22-25, fev. 2006.

TEIXEIRA, M. M. Influencia del volumen de caldo y de La uniformidad de distribución transversal sobre la eficacia de La pulverización hidráulica. 1997. Tese (Doutorado) - Universidad Politécnica de Madrid, Madrid.

VILLAS BOAS, G. L.; GAZZONI, D. L.; OLIVEIRA, M. C. N.; COSTA, N. P.; ROESSING, A. C.; HENNING, A. A. Efeito de diferentes populações de percevejos sobre o rendimento e seus componentes, características agronômicas e qualidade de semente de soja. Londrina: EMBRAPA-CNPSo, 1990. 43 p. (Boletim de Pesquisa, $1)$. 
